with cord haemorrhage. There was no evidence of vascular malformations on imaging and the screen for inflammatory myelitides was negative. A urine drug screen tested positive for sympathomimetic amines and the patient acknowledged ingesting a pill of 'unknown identity'.

Conclusion This case highlights a previously unreported complication of recreational sympathomimetics associated with significant patient morbidity. Unfortunately, this woman failed to make significant improvements during admission with ongoing severe motor and sensory deficits of her lower limbs.

\section{ENDOVASCULAR CLOT RETRIEVAL BEYOND 24 HOURS FOR TOP OF THE CAROTID OCCLUSION}

${ }^{1}$ Paul Kopanidis*, 'Shaun Zhai, ${ }^{2}$ Shivendra Lalloo, 1,3 Ronak Patel, 1,3Yash Gawarikar. ${ }^{1}$ Neurology, Calvary Public Hospital, Bruce, ACT, Australia; ${ }^{2}$ Radiology, The Canberra Hospital, Canberra, ACT, Australia; ${ }^{3}$ Medical School, Australian National University, Canberra, ACT, Australia

\subsection{6/jnnp-2019-anzan.95}

Introduction Sub-clinoid proximal occlusion is defined by internal carotid artery (ICA) occlusion with intact Circle of Willis flow. We hypothesise that such cases of large vessel occlusion provide collateral blood flow to preserve the ischaemic penumbra and may benefit from endovascular clot retrieval (ECR) beyond 24 hours.

Method We retrospectively searched the stroke database from 2018 at Calvary Hospital, Canberra, Australia for ECR cases performed beyond 24 hours from symptom onset.

Results Two patients were identified from the registry data.

64-year-old man awoke with left hemiparesis and was last seen well 9.5 hours prior. ECR for ICA occlusion was not initially performed due to rapidly improving National Institute of Health Stroke Scale (NIHSS) of three to zero. ECR was later performed at 38.5 hours for clinical deterioration. Stroke aetiology was atrial fibrillation. At 90-day NIHSS and modified Rankin Scale (mRS) were three.

75 -year-old man awoke with left hemiparesis and was last seen well 10 hours prior. Baseline NIHSS was four. Off-label thrombolysis was administered based on salvageable penumbra on CTP, however ECR for ICA occlusion was not performed as neurointervention was unavailable. After 24 hours his NIHSS score improved to one but hemispheric hypoperfusion persisted on CTP. At 36 hours he underwent ECR with carotid stenting. Stroke aetiology was large-vessel atherosclerosis. At 90 days his NIHSS and mRS were zero.

Conclusion Acute sub-clinoid proximal carotid occlusion requires tissue viability assessment with imaging to guide decision of ECR beyond 24 hours and may be of benefit.

\section{SEVERE DYSAUTONOMIA IN NMDAR ENCEPHALITIS}

${ }^{1}$ Alana Donaldson*, ${ }^{1}$ Abhay Venkat*, ${ }^{1,2}$ Shaun Zhai, ${ }^{1,2}$ Ronak Patel, ${ }^{1,2}$ Yash Gawarikar. ${ }^{1}$ Calvary Public Hospital Bruce, Canberra, ACT, Australia; ${ }^{2}$ Australian National University, Canberra, ACT, Australia

10.1136/jnnp-2019-anzan.96

Introduction Anti-N-methyl-D-aspartate receptor (NMDAR) encephalitis is one of the more common forms of autoimmune encephalitis, predominantly affecting children and women of the child-bearing age. It is characterised by memory deficit, behavioural disturbance and seizures. Dysautonomia is recognised as a feature but rarely the first symptom of the condition. ${ }^{1}$ Here we present a case of severe dysautonomia preceding the diagnosis of NMDAR encephalitis.

Methods We conducted a retrospective review of the admissions to the neurology ward at the Calvary Hospital, Canberra in 2018 to identify patients diagnosed with NMDAR encephalitis.

Results One patient was identified from the registry data. A 37-year-old woman presented with a week-long history of symptomatic orthostatic hypotension. Her supine systolic blood pressure was $110 \mathrm{mmHg}$ with a $46 \mathrm{mmHg}$ postural drop. Over the first week of hospitalisation, she became increasingly disoriented and erratic in behaviour with fluctuating levels of consciousness requiring intensive unit care. Her CSF demonstrated lymphocytic pleocytosis and NMDAR antibodies were detected in both CSF and serum. She was treated with IVIG, IV steroids and subsequently Rituximab. A pelvic teratoma was found and removed. Her symptomatology including dysautonomia improved substantially by the end of her six-week hospital admission. Her modified Rankin Scale was zero at three months.

Conclusion Autonomic dysfunction is not a common feature of autoimmune encephalitides. Our case highlighted the possibility that dysautonomia can be the initiating symptom of this disease entity. Physician awareness is important in the early recognition and treatment of this condition.

\section{REFERENCE}

1. Titulaer MJ, McCracken L, Gabilondo I, Armangue T, Glaser C, lizuka T, Honig LS, Benseler SM, Kawachi I, Martinez-Hernandez E, et al. Treatment and prognostic factors for long-term outcome in patients with anti-NMDA receptor encephalitis: an observational cohort study. Lancet Neurol 2013;12 (2):157-165.

\section{THE BENEFITS OF A CLINICAL TRIAGE TOOL IN ACUTE STROKE}

Khaled Alanati*, Sean Byrnes, James Evans, David Campbell, Ellen Wall. Department of Neuroscience, Gosford Hospital, Gosford, NSW, Australia

\subsection{6/jnnp-2019-anzan.97}

Introduction The advances in management of ischemic stroke and the extended treatment window have greatly increased the demand for acute stroke assessment. There is a need for fast and accurate triage both to identify candidates for acute stroke assessment and to minimize cost and time expended on unnecessary assessments and investigations.

We examined the potential benefit of a web-based triage tool designed to be used in the emergency department to identify patients suitable for hyperacute treatment based on the current standard of care.

Methods We performed a retrospective analysis of Electronic medical records of 235 patients, reviewed by the neurology team at Gosford Hospital for the consideration of hyperacute treatment over six months in 2017. Each patient's time of onset of symptoms, premorbid function and presenting deficit, as well as the type of treatment received, were collected. By entering those data to our new triage system, we estimated the number of clinical reviews and advanced imaging that could have been avoided. 
Results Out of the 235 patients that were reviewed, 71 patients either received thrombolysis and/or were sent for endovascular treatment, and 164 patients were not suitable for hyperacute treatment. Using the triage tool, we identified that $26 \%(n=61)$ of the rapid clinical assessment and 32\% $(n=42)$ of CT perfusion scans performed could have been avoided. Conclusion Use of a web-based triage tool is not only effective to identify patients suitable for hyperacute management but also to avoid over-investigation and prioritize rapid neurological clinical assessments.

\section{RECURRENT HEADACHES WITH PSYCHOSIS, CSF LYMPHOCYTOSIS, VESSEL BEADING AND PAPILLOEDEMA- AUTOIMMUNE/VIRAL ENCEPHALITIS WITH VASCULOPATHY OR UNUSUAL PRESENTATION OF REVERSIBLE CEREBRAL VASOCONSTRICTION SYNDROME (RCVS)?}

${ }^{1}$ Christopher J Coppin ${ }^{*},{ }^{2}$ Michael Barnett, ${ }^{3}$ Lynette Masters, ${ }^{4}$ Amjed Aziz, ${ }^{5}$ Manisha Narasimhan*. 'Basic Physician Trainee, St George and Sutherland Hospitals, Sydney, NSW, Australia; ${ }^{2}$ Department of Neurology, Royal Prince Alfred Hospital; Brain and Mind Centre (USyd), Sydney, NSW, Australia; ${ }^{3}$ Neuroradiologist, IMED Radiology, Sydney, NSW, Australia; ${ }^{4}$ Department of Critical Care Medicine, Sutherland Hospital, Sydney, NSW, Australia; ${ }^{5}$ Department of Neurology, Sutherland Hospital, Sydney, NSW, Australia

\subsection{6/jnnp-2019-anzan.98}

Introduction Headache is a common Neurology presentation in both outpatient and ED settings. We present a challenging headache in a previously asymptomatic young female patient. Case A 27 year old female patient presented to ED with severe headache, vomiting and photophobia, after multiple presentations elsewhere, diagnosed as migraine, with normal neurological examination and MRI. Episodes of excruciating headache were associated with writhing and vomiting but spontaneously resolved with residual background pain. Indomethacin helped but she re-presented with headache and psychosis requiring intubation and ICU admission.

Investigations included CSF - protein 0.85 , leucocytosis 58 cells (lymphocytic) and normal cerebral venography. Acyclovir was commenced but viral PCRs were negative and she was extubated. MRI was suspicious for subtle posterior sulcal hyperintensity and beading in occipital and posterior cerebral arteries.

Another episode occurred ten days later requiring intubation, examination showed papilloedema. CSF examination showed raised pressure, normal protein and 48 lymphocytes; flow cytometry and cytology were unrevealing. NMDA receptor antibody returned positive in serum but not CSF. Subtle beading in right PCA branches remained. IVIG and steroids were used with dramatic response. Further investigation revealed positive EBV serology only and papilloedema resolved. Steroids were weaned and she remains in remission.

Conclusion We present an interesting case of recurrent acute headaches with intracranial hypertension, psychosis and CSF lymphocytosis. The recurrent headaches and vessel beading suggest RCVS, perhaps triggered by viral or autoimmune encephalitis. The NMDA receptor antibody result should be interpreted with caution given the absence of antibody in CSF and dramatic recovery.

\section{AXONAL POLYNEUROPATHY WITH ONSET IN YOUNG ADULTHOOD DUE TO TUBB3 MUTATION}

Po Sheng Yang* ${ }^{*}$ Kimberley K Forrest, Ian B Wilson. Neurology, Cairns Base Hospital, Cairns, QLD, Australia

\subsection{6/jnnp-2019-anzan.99}

Introduction The TUBB3 gene encodes the protein Beta-tubulin isotype III, a component of the microtubule cytoskeleton. Mutations in this gene have been associated with axonal polyneuropathy, however usually associated with congenital fibrosis of the extraocular muscles (CFOEM) and other abnormalities of cerebral development. ${ }^{1} 2$ We report a case of isolated neuropathy associated with a TUBB3 mutation.

Methods Case report - clinical information and next generation sequencing results were obtained.

Results A 64 year old man presented with a severe, progressive, length dependent sensorimotor polyneuropathy which commenced in his late twenties. There was no clinical involvement of the extraocular muscles and cognition was normal. Family history was limited, but there were no other members affected.

The patient had previously been extensively investigated including sural nerve biopsy, which confirmed axonal neuropathy without a specific diagnosis. Intravenous immunoglobulins and steroids had been trialled without benefit.

A neuromuscular gene panel utilising next generation sequencing was performed and demonstrated heterozygosity for a variant of the TUBB3 gene (D417N substitution).

Case series describing TUBB3 mutations show a large heterogeneity in phenotypic expression depending on the amino acid substitution. ${ }^{2-4}$ There is also heterogeneity in patients with $\mathrm{D} 417 \mathrm{~N}$ mutations, although a small number have been reported to develop a polyneuropathy without CFOEM $^{1}$.

Conclusions This case strengthens previous reports that TUBB3 mutation can be associated with a pure, axonal, sensorimotor polyneuropathy and highlights the use of next generation sequencing in streamlining the diagnostic process.

\section{REFERENCE}

1. Tischfield $\mathrm{M}$, et al. Human TUBB3 mutations perturb microtubule dynamics, kinesin interactions, and axon guidance. Cell 2010;140(1):pp.74-87.

2. Ncbi.nlm.nih.gov. (2019). Congenital fibrosis of the extraocular muscles - Conditions - GTR - NCBI. [online] Available at: https://www.ncbi.nlm.nih.gov/gtr/conditions/CN043677/ [Accessed 14 Feb. 2019].

3. Omim.org. (2019). OMIM Entry - *602661 - TUBULIN, BETA-3; TUBB3. [online] Available at: https://www.omim.org/entry/602661 [Accessed 14 Feb. 2019].

4. Krupa, K. and Bekiesinska-Figatowska, M. (2013). Congenital and Acquired Abnormalities of the Corpus Callosum: A Pictorial Essay. BioMed Research International, 2013, pp.1-14

\section{A CASE OF SUSPECTED AUTOIMMUNE ENCEPHALITIS SECONDARY TO NIVOLUMAB}

${ }^{1}$ Jaimie Ho*, ${ }^{2}$ Yun Hwang, ${ }^{3}$ Martin Tio, ${ }^{3}$ Georgina Long, ${ }^{4}$ Eva Zhang. ${ }^{1}$ Gosford Hospital, Gosford, NSW, Australia; ${ }^{2}$ Neurology, Gosford Hospital, Gosford, NSW, Australia; ${ }^{3}$ Melanoma Institute Australia, Sydney, NSW, Australia; ${ }^{4}$ Royal North Shore Hospital, Sydney, NSW, Australia

\subsection{6/jnnp-2019-anzan.100}

Objective To describe a case of suspected immune encephalitis following nivolumab for metastatic melanoma. 